\title{
The Fat but Fit paradox: what we know and don't know about it
}

\author{
Francisco B Ortega, ${ }^{1}$ Jonatan R Ruiz, ${ }^{1}$ Idoia Labayen, ${ }^{2}$ Carl J Lavie, ${ }^{3}$ \\ Steven N Blair ${ }^{4,5}$
}

\section{WHAT WE KNOW ABOUT IT}

In the late $1980 \mathrm{~s}$, one of us (SNB) published a study that demonstrated that individuals with a low (below first quintile $=20$ th percentile) cardiorespiratory fitness level had a higher risk of mortality 8 years later, when compared with those who were at least moderately fit. ${ }^{1}$ Since then, many longitudinal studies have consistently confirmed this notion in men and women, as well as in healthy and diseased individuals, for all-cause mortality, as well as for cardiovascular disease (CVD) mortality. ${ }^{2}$

Obesity is related to multiple physical and mental comorbidities and it is an incontrovertible risk factor for all-cause and CVD mortality. ${ }^{3}$ It has been suggested, however, that being fit might attenuate some of the adverse consequences of obesity, independently of some key potential confounders. In this context, in the late 1990s, some studies provided first evidence for what was later known as the Fat but Fit paradox (see review by Ortega et $a l .{ }^{3}$ ). These studies demonstrated that all-cause and CVD mortality risk in obese individuals, as defined by body mass index (BMI), body fat percentage or waist circumference, who are fit (ie, cardiorespiratory fitness level above the age-specific and sex-specific 20th percentile) is not significantly different from their normal-weight and fit counterparts (ie, the theoretically healthiest group possible) (figure 1).

\section{Being normal-weight might not be enough: being fit plays a major role in health}

There is a general belief that being normalweight is synonymous to being healthy, yet this notion could be wrong. In many cases, normal-weight but unfit individuals have significantly higher risk of all-cause and CVD mortality than normal-weight fit individuals. In addition, some readers will find even more interesting the fact that several studies have reported that normalweight but unfit individuals could be at a higher risk than obese but fit individuals (figure 1), which might seem paradoxical. ${ }^{3}$
Role of genes and physical activity

As for many other phenotypes, genes and environment jointly influence both adiposity and cardiorespiratory fitness. Heritability of both obesity and cardiorespiratory fitness might be up to $50 \%$ (eg, results from the HEalth, RIsk factors, exercise Training And GEnetics (HERITAGE) Family Study led by Professor Bouchard and colleagues), leaving environmental factors to explain the rest of the variance. Among environmental factors, regular physical activity (PA) and, particularly, that of vigorous intensity have shown to be the most effective in improving cardiorespiratory fitness. For more detailed information about how to improve cardiorespiratory fitness through PA, see tables 7 and 8 from the recently published Scientific Statement from the American Health Association. ${ }^{2}$

\section{WHAT WE STILL DON'T KNOW ABOUT IT}

There is emerging evidence suggesting that a moderate to high cardiorespiratory fitness might counteract the negative consequences of obesity on many other health outcomes, especially in certain population age groups, such as children and adolescents. Below, we highlight potential research directions for future studies.
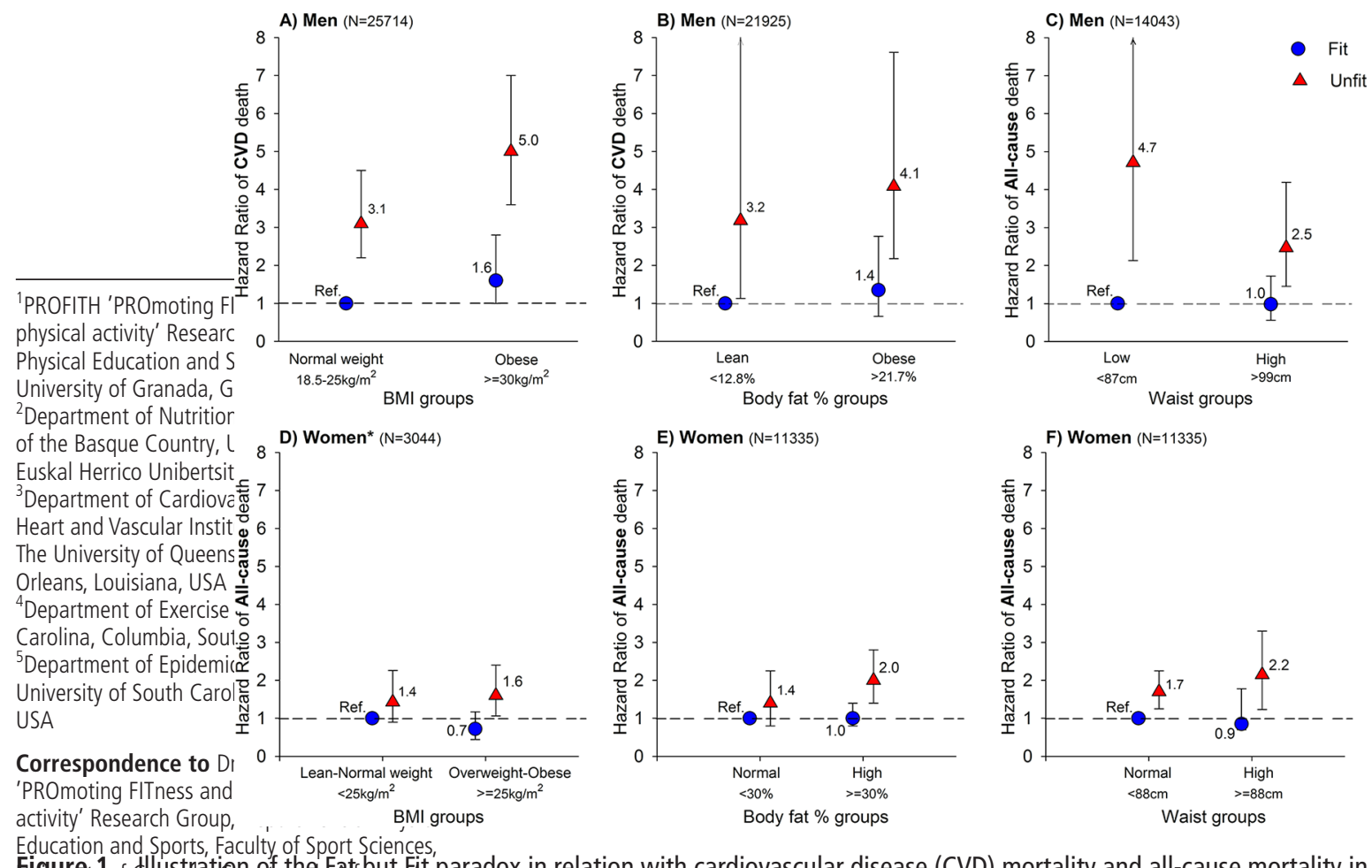

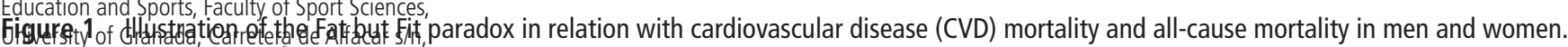

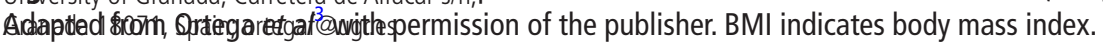


Table 1 Cardiorespiratory fitness (maximal oxygen consumption, V02max, $\mathrm{mL} / \mathrm{kg} / \mathrm{min}$ ) cut-points to classify individuals as unfit

\begin{tabular}{|c|c|c|c|c|}
\hline \multicolumn{5}{|c|}{ An individual will be classified as unfit if his/her $\mathrm{VO}_{2} \mathrm{max}^{*}$ is below these cut-points, and as fit otherwise } \\
\hline \multirow{2}{*}{$\begin{array}{l}\text { Age group } \\
\text { (years) }\end{array}$} & \multicolumn{2}{|c|}{ Treadmill test } & \multicolumn{2}{|l|}{ Bike test } \\
\hline & Boys/men & Girls/women & Boys/men & Girls/women \\
\hline $8-19+$ & 42.0 & 35.0 & 42.0 & 35.0 \\
\hline $20-29$ & 38.1 & 28.6 & 33.2 & 21.6 \\
\hline $30-39$ & 34.1 & 24.1 & 25.4 & 17.0 \\
\hline 40-49 & 30.5 & 21.3 & 22.2 & 15.8 \\
\hline 50-59 & 26.1 & 19.1 & 21.5 & 14.9 \\
\hline $60-69$ & 22.4 & 16.5 & 19.0 & 14.0 \\
\hline $70+$ & 19.2 & 15.1 & 16.7 & 12.8 \\
\hline
\end{tabular}

* It is important to note that heavier individuals could bepenalised when cardiorespiratory fitness is expressed as VO $\mathrm{max}$, $\mathrm{mL} / \mathrm{kg} / \mathrm{min}$. In these cases, alternatively, total treadmill time in an incremental maximal exercise test could be used as indicator of fitness level, for which the reference values of the Cooper Institute can be used (Physical Fitness Assessments and Norms for Adults and Law Enforcement; Cooper Institute; 2013).

tThe cut-points for individuals aged 8 to 19 years come from a meta-analysis recently published by Ruiz et al. ${ }^{4}$ In studies in which the 20 m shuttle run test was used to assess cardiorespiratory fitness in children or adolescents, an alternative definition of unfit can be done using thefirst quintile of the international reference value derived from the recent meta-analysis published by Tomkinson et al. ${ }^{5}$

The cut-points for adults correspond to the sex-specific and age-specific first quintile from the data (2014-2015) from 7783 maximal (respiratory exchange ratio $\geq 1.0$ ) treadmill tests from men and women (aged 20-79 years) without cardiovascular disease from the Fitness Registry and the Importance of Exercise: A National Data Base (FRIEND). ${ }^{9}$ Equivalent reference values derived from maximal bike tests, also from FRIEND, are provided $(n=4494) .{ }^{10}$ To our knowledge, these are the most accurate, largest and more updated fitness reference values covering all the adult age groups to date.

\section{The Fat but Fit paradox in children and adolescents}

Our recent meta-analysis demonstrated a strong link between cardiorespiratory fitness and cardiometabolic risk factors in children and adolescents, providing cut-points that more accurately delineate CVD risk (derived from receiver operating characteristic analyses). ${ }^{4}$ Worldwide reference values (sex specific and age specific) for cardiorespiratory fitness have also been recently published. ${ }^{5}$ These two meta-analyses have major practical implications as they allow children's cardiorespiratory fitness level to be properly interpreted and ranked. Specific information about the Fat but Fit paradox in young populations is scarce; yet, there is evidence suggesting that having moderate to high levels of cardiorespiratory fitness may attenuate the deleterious metabolic consequences ascribed to an excess total and central adiposity. $^{67}$

\section{The Fat but Fit paradox and other health outcomes}

Fat but Fit individuals have a 50\% reduction in the risk of developing depression compared with their Fat and Unfit peers. ${ }^{8}$ It is very likely that fitness and fatness counteract in their association with many physical and mental health outcomes, such as psychiatric disorders (eg, attention deficit hyperactivity disorder), self-esteem, cancer, disability pension, heart rate variability, hepatic steatosis (fatty liver), brown adipose tissue volume and activity, cognition, and brain structure and function.

\section{The Fat but Fit paradox in randomised controlled trials}

Please note that all evidence available for the Fat but Fit paradox comes from observational studies. Consequently, even if most of the studies are prospective cohorts and their findings provide highly valuable prognostic information, causeeffect relationships cannot be confirmed. Although exercise interventions in obese individuals lowered CVD risk, even without weight loss, literature is limited regarding whether these improvements in health without weight loss are driven by increases in cardiorespiratory fitness. In other words, such studies will be able to test the Fat but Fit paradox using an experimental design. Future exercise-based randomised controlled trials conducted in obese population should therefore include an accurate assessment of cardiorespiratory fitness before and after intervention so that formal mediation analyses can be performed.

\section{TAKE-HOME MESSAGE}

Obesity, and especially severe/morbid obesity $\left(\mathrm{BMI}>35 \mathrm{~kg} / \mathrm{m}^{2}\right)$, is a major public health problem. Lifestyle intervention programs for obesity with special focus on exercise and a healthy diet are important public health goals. However, focus should not be placed exclusively on losing weight/fat, but also on increasing cardiorespiratory fitness, since a medium-high cardiorespiratory fitness level may attenuate the adverse consequences of obesity on health. The information summarised in this Editorial support that future public health strategies planned for obese individuals should target, in parallel, both weight/fat reduction and cardiorespiratory fitness improvement, especially if the obese person is classified as unfit, that is, below the sex-specific and age-specific 20th percentile.

Table 1 will help sports specialists, physicians and other healthcare practitioners to classify individuals as unfit versus fit, which together with the internationally accepted definition of obesity $\left(\mathrm{BMI} \geq 30 \mathrm{~kg} / \mathrm{m}^{2}\right)$ results in the definition of Fat but Fit.

Contributors FBO wrote the initial draft of the article, which was then discussed and refined with the other authors.

Funding FBO was supported by the Spanish Ministry of Economy and Competitiveness - MINECO (RYC-2011-09011). Additional support was obtained from the MINECO/FEDER (DEP2013-47540-R and DEP2016-79512-R); the University of Granada, Plan Propio de Investigación 2016, Excellence actions: Units of Excellence, Unit of Excellence on Exercise and Health (UCEES); the European Union's Horizon 2020 research and innovation programme under grant agreement no. 667302; the SAMID III network, RETICS, funded by the PN I+D+I 2017-2021 (Spain), ISCIII-Sub-Directorate General for Research Assessment and Promotion, the European Regional Development Fund (ERDF) (Ref. RD16/0022) and the EXERNET Research Network on Exercise and Health in Special Populations (DEP2005-00046/ACTI). CJL and SNB has received unrestricted research grants from Coca-Cola Company.

Disclaimer The views expressed are those of the authors and do not reflect the official policy or position of the institutions they belong to.

Competing interests $\mathrm{CJL}$ has served as a consultant and speaker on fitness/obesity for the Coca-Cola and has published a book on the obesity paradox with potential royalties. SNB has served as consultant for 
weight loss and fitness companies and for the CocaCola.

Provenance and peer review Not commissioned; externally peer reviewed.

(c) Article author(s) (or their employer(s) unless otherwise stated in the text of the article) 2018. All rights reserved. No commercial use is permitted unless otherwise expressly granted.

\section{A) Check for updates}

To cite Ortega FB, Ruiz JR, Labayen I, et al. Br J Sports Med 2018;52:151-153.

Accepted 28 April 2017

Published Online First 5 June 2017

Br J Sports Med 2018;52:151-153.

doi:10.1136/bjsports-2016-097400

\section{REFERENCES}

1 Blair SN, Kohl HW, Paffenbarger RS, et al. Physical fitness and all-cause mortality. A prospective study of healthy men and women. JAMA 1989;262:2395-401.

2 Ross R, Blair SN, Arena R, et al. Importance of assessing cardiorespiratory fitness in clinical practice: A case for fitness as a clinical vital sign: A scientific statement from the American Heart Association. Circulation 2016;134:e653-e699.

3 Ortega FB, Lavie CJ, Blair SN. Obesity and Cardiovascular Disease. Circ Res 2016;118:1752-70.

4 Ruiz JR, Cavero-Redondo I, Ortega FB, et al. Cardiorespiratory fitness cut points to avoid cardiovascular disease risk in children and adolescents; what level of fitness should raise a red flag? A systematic review and meta-analysis. Br I Sports Med 2016:3:1451-8

5 Tomkinson GR, Lang JJ, Tremblay MS, et al. International normative $20 \mathrm{~m}$ shuttle run values from 1142026 children and youth representing 50 countries. Br J Sports Med 2017;51:1545-54.
6 DuBose KD, Eisenmann JC, Donnelly JE. Aerobic fitness attenuates the metabolic syndrome score in normal-weight, at-risk-for-overweight, and overweight children. Pediatrics 2007:120:e1262-e1268.

7 Mesa JL, Ruiz JR, Ortega FB, et al. Aerobic physical fitness in relation to blood lipids and fasting glycaemia in adolescents: influence of weight status. Nutr Metab Cardiovasc Dis 2006:16:285-93.

8 Becofsky KM, Sui X, Lee DC, et al. A prospective study of fitness, fatness, and depressive symptoms. Am J Epidemiol 2015;181:311-20.

9 Kaminsky LA, Arena R, Myers J. Reference standards for cardiorespiratory fitness measured with cardiopulmonary exercise testing: data from the fitness registry and the importance of exercise national database. Mayo Clin Proc 2015;90:1515-23.

10 Kaminsky LA, Imboden MT, Arena R, et al. Reference standards for cardiorespiratory fitness measured with cardiopulmonary exercise testing using cycle ergometry: data from the Fitness Registry and the Importance of Exercise National Database (FRIEND) Registry. Mayo Clin Proc 2017;92:228-33. 